\title{
Author Correction: Isolation and cultivation of naive-like human pluripotent stem cells based on HERVH expression
}

Jichang Wang, Manvendra Singh, Chuanbo Sun, Daniel Besser, Alessandro Prigione, Zoltán Ivics, Laurence D Hurst, Zsuzsanna Izsvák

Correction to: Nature Protocols https://doi.org/10.1038/nprot.2016.016, published online 21 January 2016

In the published version of this paper, the authors omitted a funding source. L.D.H. acknowledges support from the European

Research Council (Advanced Grant ERC-2014-ADG 669207). The original article has not been corrected.

Published online: 29 October 2018

https://doi.org/10.1038/s41596-018-0086-6

\section{Publisher Correction: Acute and rapid degradation of endogenous proteins by Trim-Away}

Dean Clift, Chun So, William A. McEwan, Leo C. James, Melina Schuh

Correction to: Nature Protocols https://doi.org/10.1038/s41596-018-0028-3, published online 24 September 2018

In the version of this paper originally published, the present address of W.A. McEwan was accidentally omitted. This address (UK Dementia Research Institute, Department of Clinical Neurosciences, University of Cambridge, Cambridge, UK) has now been added as affiliation 3, and the equal-contributions note has been updated to affiliation 4 . These changes are reflected in the PDF and HTML versions of the protocol.

Published online: 30 November 2018

https://doi.org/10.1038/s41596-018-0092-8

\section{Publisher Correction: PhIP-Seq characterization of serum antibodies using oligonucleotide-encoded peptidomes}

Divya Mohan, Daniel L. Wansley, Brandon M. Sie, Muhammad S. Noon (D) Alan N. Baer, Uri Laserson,

H. Benjamin Larman

Correction to: Nature Protocols https://doi.org/10.1038/s41596-018-0025-6, published online 6 September 2018

The version of this paper originally published contained typesetter-introduced errors in some of the code commands, consisting of conversion of a closing backslash $(\backslash)$ to a forward slash (/). These errors have been corrected in the HTML and PDF versions of the protocol.

Published online: 25 October 2018

https://doi.org/10.1038/s41596-018-0088-4 